\title{
ULTRASTRUCTURAL AND BIOCHEMICAL STUDIES ON SUBCELLULAR FRACTIONS OF CHLOROQUINE INDUCED AUTOPHAGIC VACUOLES*
}

\author{
Michael J. Brabec, Roberta K. Brabec and Robert H. Gray \\ Department of Environmental and Industrial Health, Environmental Cellular Chemistry Laboratory, \\ The University of Michigan, Ann Arbor, MI 48109, U.S.A.
}

Subcellular fractions enriched for autophagic vacuoles (AV) (Fig. 1) have been isolated from rat hepatocytes $2 \mathrm{hr}$ following the administration of chloroquine (Gray et al., 1973). This study further characterizes the subcellular fractions ultrastructurally and biochemically in addition to following the distribution of ${ }^{14} \mathrm{C}$-chloroquine in the subcellular fractions. Fractions were assayed for their protein content and the specific activities of a lysosomal enzyme, acid phosphatase (AcPase), and two microsomal enzymes, glucose-6-phosphatase (G6Pase) and TPNHDCIP reductase. Two AV-enriched fractions, B-2 and B-3, were recovered from the final discontinuous (B) gradient. The fractions differ with respect to the degree of degradation of the components sequestered within the AV. The AV of the B-2 fraction (Figs. 2 and 3) primarily contain concentric membranes with relatively few recognizable organelles, whereas the AV in the B-3 (Fig. 4) fraction contain some identi-

\footnotetext{
* Work supported by Michigan Memorial Phoenix Project Grant No. 478.
}

fiable organelles (mitochondria and peroxisomes) and fewer layers of concentric membranes. The distribution of protein, AcPase and G6Pase are essentially parallel in their distribution on the final discontinuous gradient. The TPNH-DCIP reductase content was too low to be measured in the final fractions. The ${ }^{14} \mathrm{C}$-chloroquine also shows high incorporation levels in the B-2 and B-3 fractions as well as an additional peak in a less dense band, B-1. The results indicate that the enriched $\mathrm{AV}$ fractions contain significant amounts of AcPase. The presence of G6Pase in the enriched AV fractions supports the view that membranes of these organelles are of endoplasmic reticulum origin. The high specific activity of ${ }^{14} \mathrm{C}$-chloroquine in the B-2 and B-3 AV enriched fractions demonstrates the affinity of the AV for the drug. These observations suggest that the AV play an important role in the accommodation of subcellular stress by selectively sequestering a perturbing agent present in a cell.

\section{REFERENCES}

Gray, R. H. et al., 1973. J. Cell Biol., 59: 121a. 


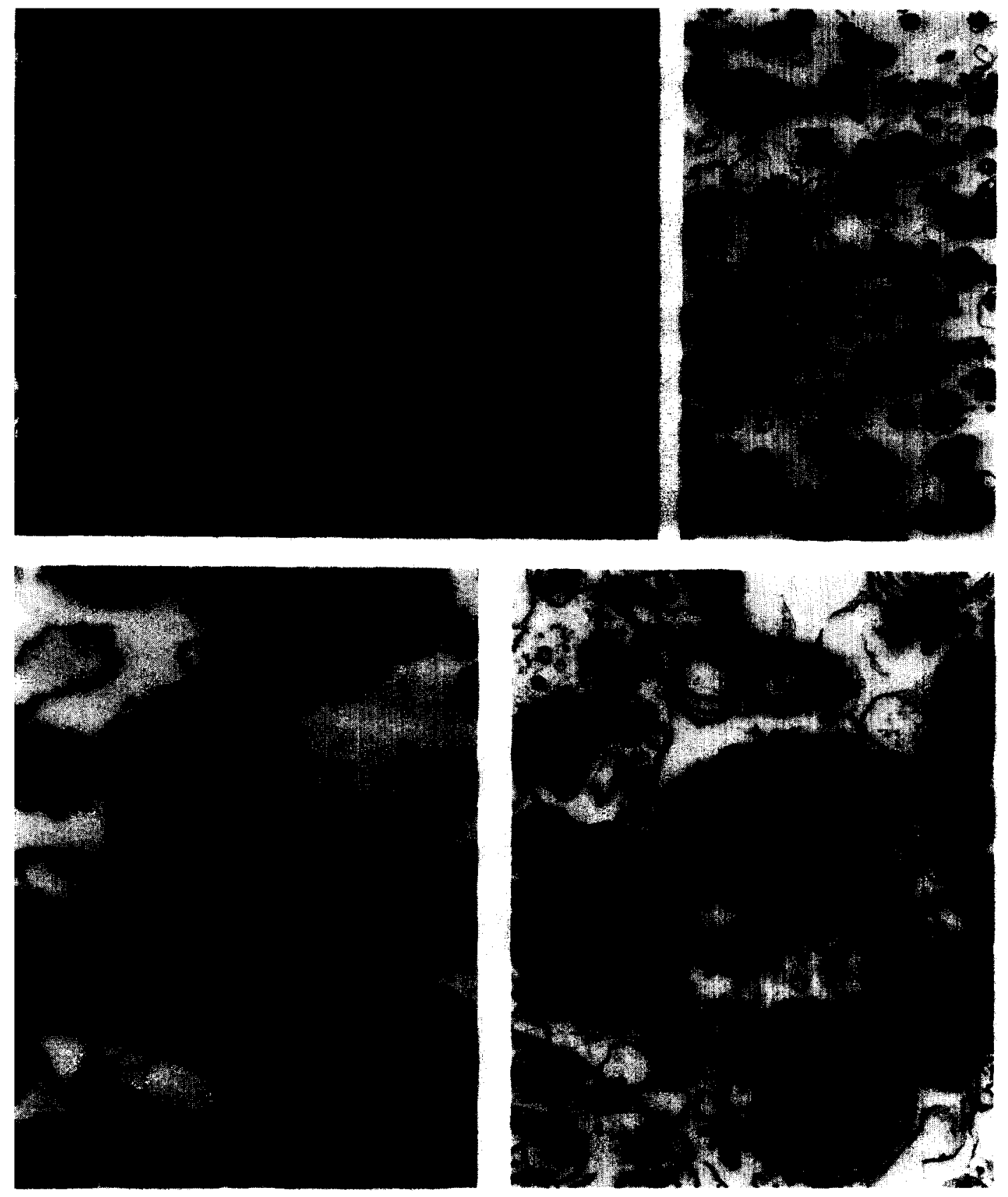

Fig. 1. Chloroquine induced hepatocyte autophagic vacuoles in situ $2 \mathrm{hr}$ after drug administration. $\times 20,000$.

Fig. 2. TEM survey view of $B-2$ fraction of autophagic vacuoles. $\times 8000$.

Figs. 3 and 4 . Isolated autophagic vacuoles from fraction $B-2$ and $B-3 \times 30,000$, , respectively. 